\title{
On Henstock Method to Stratonovich Integral with respect to Continuous Semimartingale
}

\author{
Haifeng Yang and Tin Lam Toh \\ National Institute of Education, Nanyang Technological University, Singapore 637616 \\ Correspondence should be addressed to Tin Lam Toh; tinlam.toh@nie.edu.sg \\ Received 15 September 2014; Revised 26 November 2014; Accepted 26 November 2014; Published 18 December 2014 \\ Academic Editor: Fred Espen Benth
}

Copyright ( $) 2014$ H. Yang and T. L. Toh. This is an open access article distributed under the Creative Commons Attribution License, which permits unrestricted use, distribution, and reproduction in any medium, provided the original work is properly cited.

We will use the Henstock (or generalized Riemann) approach to define the Stratonovich integral with respect to continuous semimartingale in $L^{2}$ space. Our definition of Stratonovich integral encompasses the classical definition of Stratonovich integral.

\section{Introduction}

Classically, it has been emphasized that defining stochastic integrals using Riemann approach is impossible [1, p. 40], since the integrators have paths of unbounded variation. Moreover, the integrands are usually highly oscillatory [13]. The uniform mesh in classical Riemann setting is unable to handle highly oscillatory integrators and integrands. Kurzweil and Henstock [4-6] independently modified the Riemann approach by using nonuniform mesh. This modification leads to a larger class of integrals being studied (see $[4,7-12])$.

The generalized Riemann approach, using nonuniform meshes, has been successful in giving an alternative definition to the Stratonovich integral with respect to Brownian motion (see [13]). This paper attempts to further generalize the result of [13] to define the Stratonovich integral with respect to continuous semimartingale.

The difficulty to extend from Brownian motion to semimartingale is that the quadratic variation of the continuous martingale may not be absolutely continuous. Hence, the definition of the Stratonovich-Henstock belated integral in [13] may not be valid. In [12, 14, 15], the definition of weakly Henstock variation belated integral was used to address this difficulty. Similarly, we tap on this approach to define Stratonovich integral with respect to continuous $L^{2}$ martingale.
Further, to consider continuous local martingale as an integrator, we will show that a $(\delta, \eta)$-fine belated partial stochastic division $D=\left\{\left(I_{k}, \xi_{k}\right)\right\}_{k=1}^{n}$ of $[0, t]$ in $[12,16]$ is also a $(\delta, \eta)$-fine belated partial stochastic division of $[0, t \wedge S]$, where $S$ is a stopping time.

Lastly, the definition of the Stratonovich integral with respect to continuous semimartingale will be further refined. We divide the continuous semimartingale into two parts: a continuous local martingale and the difference of continuous, nondecreasing, and adapted processes. The former is discussed in Section 3.2. The latter has finite total variation on each bounded interval. Hence, integration with respect to the latter process is classical Riemann-Stieltjes. This part has already been addressed in [17].

We give a new definition of the Stratonovich integral with continuous semimartingale in $L^{2}$ space. This integrand is weaker than the classical case.

\section{Stratonovich Integral}

In this section, we will generalize the definition of Stratonovich integral to include the case where the integrator is continuous $L^{2}$-semimartingale. We will develop the definition of Stratonovich-Henstock belated integral where the integrator is a Brownian motion in [13]. Let $(\Omega, \mathscr{F}, P)$ be a probability 
space such that $(\Omega, \mathscr{F}, P)$ is complete. Also, let $C^{n}(\mathbb{R})$ denote the class of functions which have continuous $n$ derivatives.

Definition 1. Let $X=\left\{X_{t}, \mathscr{F}_{t} ; 0 \leq t<\infty\right\}$ be a process. If there exists a nondecreasing sequence $\left\{T_{n}\right\}_{n=1}^{\infty}$ of the stopping times of the filtration $\left\{\mathscr{F}_{t}\right\}$, such that $\left\{X_{t}^{(n)} \triangleq X_{t \wedge T_{n}}, \mathscr{F}_{t} ; 0 \leq\right.$ $t<\infty\}$ is a martingale for each $n \geq 1$ and $P\left[\lim _{n \rightarrow \infty} T_{n}=\right.$ $\infty]=1$, then one says that $X$ is local martingale. If, in addition, $X_{0}=0$ a.s., one writes $X \in \mathscr{M}^{\text {loc }}$ (or $X \in \mathscr{M}^{\text {, loc }}$, if $X$ is continuous).

Definition 2. A continuous semimartingale $X=\left\{X_{t}, \mathscr{F}_{t} ; 0 \leq\right.$ $t<\infty\}$ is an adapted process which has the decomposition

$$
X_{t}=X_{0}+M_{t}+C_{t}, \quad 0 \leq t<\infty \text { a.s., }
$$

where $M=\left\{M_{t}, \mathscr{F}_{t} ; 0 \leq t<\infty\right\} \in \mathscr{M}^{\text {, loc }}$ and $C_{t}$ has finite variation.

Definition 3. Let $X=\left\{X_{t}, \mathscr{F}_{t} ; 0 \leq t<\infty\right\}$ and $Y=$ $\left\{Y_{t}, \mathscr{F}_{t} ; 0 \leq t<\infty\right\}$ be two continuous semimartingales such that

$$
X_{t}=X_{0}+M_{t}+C_{t} ; \quad Y_{t}=Y_{0}+N_{t}+D_{t},
$$

where $M$ and $N$ are in $\mathscr{M}^{\text {, loc }}$ and $C_{t}$ and $D_{t}$ are adapted, continuous process of bounded variation with $C_{0}=D_{0}=0$ a.s. The Stratonovich integral of $Y_{t}$ with respect to $X_{t}$ is

$$
\int_{0}^{t} Y_{s} \circ d X_{s} \triangleq \int_{0}^{t} Y_{s} d M_{s}+\int_{0}^{t} Y_{s} d C_{s}+\frac{1}{2}\langle M, N\rangle_{t},
$$

where the first integral on the right-hand side of (3) is an Itô integral, the second one is pathwise integral of the RiemannStieltjes type, and $\langle M, N\rangle_{t}$ is the cross variation process of $M$ and $N$. If $M=N$, then $\langle M, N\rangle_{t}=\langle M\rangle_{t}$ is the quadratic variation of $M$ (see [18, p. 31]).

Proposition 4. Let $f: \mathbb{R} \rightarrow \mathbb{R}$ be a function of class $C^{3}(\mathbb{R})$ and let $X=\left\{X_{t}, \mathscr{F}_{t} ; 0 \leq t<\infty\right\}$ be a continuous semimartingale with decomposition in (2). Then

$$
f\left(X_{t}\right)=f\left(X_{0}\right)+\int_{0}^{t} f^{\prime}\left(X_{s}\right) \circ d X_{s} \text { a.s. }
$$

Proof. By Itô formula [18, p. 149], we have

$$
f\left(X_{t}\right)=f\left(X_{0}\right)+\int_{0}^{t} f^{\prime}\left(X_{s}\right) d X_{s}+\frac{1}{2} \int_{0}^{t} f^{\prime \prime}\left(X_{s}\right) d\langle M\rangle_{s} .
$$

We only have to prove that

$$
\int_{0}^{t} f^{\prime}\left(X_{s}\right) \circ d X_{s}=\int_{0}^{t} f^{\prime}\left(X_{s}\right) d X_{s}+\frac{1}{2} \int_{0}^{t} f^{\prime \prime}\left(X_{s}\right) d\langle M\rangle_{s} .
$$

Since $f^{\prime}\left(X_{s}\right) \in C^{2}(\mathbb{R})$, then, by Itô formula,

$$
\begin{aligned}
f^{\prime}\left(X_{s}\right)= & f^{\prime}\left(X_{0}\right)+\int_{0}^{t} f^{\prime \prime}\left(X_{s}\right) d M_{s}+\int_{0}^{t} f^{\prime \prime}\left(X_{s}\right) d A_{s} \\
& +\frac{1}{2} \int_{0}^{t} f^{\prime \prime \prime}\left(X_{s}\right) d\langle M\rangle_{s} .
\end{aligned}
$$

Then $f^{\prime}\left(X_{s}\right)$ is a semimartingale, $\int_{0}^{t} f^{\prime \prime}\left(X_{s}\right) d M_{s}$ is a local martingale, and $\int_{0}^{t} f^{\prime \prime}\left(X_{s}\right) d A_{s}$ and $(1 / 2) \int_{0}^{t} f^{\prime \prime \prime}\left(X_{s}\right) d\langle M\rangle_{s}$ are bounded continuous processes. By definition of a semimartingale,

$$
\begin{aligned}
\int_{0}^{t} f^{\prime}\left(X_{s}\right) \circ d X_{s}= & \int_{0}^{t} f^{\prime}\left(X_{s}\right) d X_{s} \\
& +\frac{1}{2}\left\langle M, \int_{0}^{t} f^{\prime \prime}\left(X_{s}\right) d M_{s}\right\rangle_{t} .
\end{aligned}
$$

By the property of cross variation (see [18, p. 143])

$$
\left\langle M, \int_{0}^{t} f^{\prime \prime}\left(X_{s}\right) d M_{s}\right\rangle_{t}=\int_{0}^{t} f^{\prime \prime}\left(X_{s}\right) d\langle M\rangle_{s} .
$$

Hence,

$$
\begin{aligned}
f\left(X_{t}\right) & =f\left(X_{0}\right)+\int_{0}^{t} f^{\prime}\left(X_{s}\right) d X_{s}+\frac{1}{2} \int_{0}^{t} f^{\prime \prime}\left(X_{s}\right) d\langle M\rangle_{s} \\
& =f\left(X_{0}\right)+\int_{0}^{t} f^{\prime}\left(X_{s}\right) \circ d X_{s} .
\end{aligned}
$$

\section{Henstock Approach}

3.1. Henstock Approach to Define Stratonovich Integral with respect to Bounded Continuous Martingale. In [13-16, 19], a positive function $\delta$ on $[0, t], 0 \leq t<\infty$, where $\delta$ does not depend on $\omega \in \Omega$, was used. From the proof of the main results in $[13,14,16,20], \delta(\xi)$ is deterministic, because the quadratic variation of a Brownian motion is deterministic and Fubini's theorem can be applied in switching the order of the two integrals. In this section, we consider martingales as integrators. To define stochastic integrals using Riemann sums, the $\delta$-function needs to depend on $\Omega$ and stochastic intervals are needed. Therefore, we need to redefine $\delta$ as also dependent on $\Omega$ (see [12]).

Definition 5. Let $\delta:[0, t] \times \Omega \rightarrow(0, t), 0 \leq t<\infty$, be a measurable function with respect to the two variables $\xi \in$ $[0, t]$ and $\omega \in \Omega$. Then $\delta$ is called a locally stopping process [12] if, for each $\xi \in[0, t], \xi+\delta$ is a stopping time.

For each $(c, d] \subset[0, t]$, we denote the measure [15] induced by the quadratic variation process of an adapted process $X=\left\{X_{t}, 0 \leq t<\infty\right\}$ by

$$
\mu_{X}(c, d] \triangleq E\left(\langle X\rangle_{d}-\langle X\rangle_{c}\right) .
$$

Definition 6. Let $\delta$ be a locally stopping process and $X=$ $\left\{X_{t}, \mathscr{F}_{t} ; 0 \leq t<\infty\right\}$. A finite collection of stochastic intervalpoint pairs $\left(\left(\left(S_{i}, T_{i}\right], \xi_{i}\right), i=1,2, \ldots, n\right)$, where $S_{i}$ and $T_{i}$ are stopping times of $X$ for all $i=1,2, \ldots, n$, is said to be a $(\delta, \eta)$ fine belated partial stochastic division of $[0, t]$ if

(1) for each $i\left(S_{i}, T_{i}\right]$ is a stochastic interval and, for each $\omega \in \Omega,\left(S_{i}, T_{i}\right], i=1,2, \ldots, n$, are disjoint left-open subintervals of $[0,1]$; 
(2) each $\left(\left(S_{i}, T_{i}\right], \xi_{i}\right)$ is a $\delta$-fine belated division; that is, for each $\omega \in \Omega$, one has

$$
\left(S_{i}(\omega), T_{i}(\omega)\right] \subset\left[\xi_{i}, \xi_{i}+\delta\left(\xi_{i}, \omega\right)\right]
$$

(3) for any $\eta>0\left|\mu_{X}(0, t]-\sum_{i=1}^{n} \mu_{X}\left(S_{i}, T_{i}\right]\right|<\eta$.

For the case $S_{i}(\omega)=T_{i}(\omega),\left(S_{i}(\omega), T_{i}(\omega)\right]$ denotes $\left\{T_{i}(\omega)\right\}$. Note that, for each $\xi \in[0, t], \xi+\delta$ is a stopping time. Thus, for each $\xi_{i}$, there exists a stochastic interval $\left(S_{i}(\omega), T_{i}(\omega)\right]$ with $S_{i}(\omega)<T_{i}(\omega)$ such that $\left(S_{i}(\omega), T_{i}(\omega)\right] \subset\left[\xi_{i}, \xi_{i}+\delta\left(\xi_{i}, \omega\right)\right]$. For each $\omega \in \Omega$, namely,

$$
S_{i}(\omega)=\xi_{i}(\omega), \quad T_{i}(\omega)=\inf \left\{\xi_{i}+\delta\left(\xi_{i}, \omega\right), t\right\} .
$$

In addition, by Vitali's covering theorem, the $(\delta, \eta)$-fine belated partial stochastic division could cover $[0, t]$ except for an arbitrarily small $\mu_{X}$-measure $[19$, p. 44$][12,16]$.

Definition 7. An adapted process $Y=\left\{Y_{t}, \mathscr{F}_{t} ; 0 \leq t<\right.$ $\infty\}$ in $(\Omega, \mathscr{F}, P)$ is said to be weakly Stratonovich-Henstock belated (denoted by WSHB) integrable to a process $A$ in $\left.\left(\Omega, \mathscr{F}_{,}, \mathscr{F}_{t}\right\}_{t \geq 0}, P\right)$ on $[0, t], 0 \leq t<\infty$, with respect to a stochastic process $X$ if, for every $\varepsilon>0$, there exists a locally stopping process $\delta$ on $[0, t] \times \Omega$ for which

$$
\begin{gathered}
E \mid \sum_{i=1}^{n}\left\{\frac{Y\left(\xi_{i+1}, \omega\right)+Y\left(\xi_{i}, \omega\right)}{2}\left(X\left(T_{i}(\omega), \omega\right)-X\left(S_{i}(\omega), \omega\right)\right)\right. \\
\left.-\left[A\left(T_{i}(\omega), \omega\right)-A\left(S_{i}(\omega), \omega\right)\right]\right\}\left.\right|^{2} \leq \varepsilon,
\end{gathered}
$$

where $\xi_{n+1}=t$. For succinctness, one may write

$$
\begin{aligned}
& E\left(\left|\sum_{i=1}^{n} \frac{Y\left(\xi_{i+1}, \omega\right)+Y\left(\xi_{i}, \omega\right)}{2}\left(X_{T_{i}}-X_{S_{i}}\right)-\left(A_{T_{i}}-A_{S_{i}}\right)\right|^{2}\right) \\
& \quad \leq \varepsilon
\end{aligned}
$$

for every $(\delta, \eta)$-fine belated partial stochastic division $D=$ $\left\{\left(\left(S_{i}, T_{i}\right], \xi_{i}\right), i=1,2, \ldots, n\right\}$ of $[0, t]$.

Proposition 8. If an adapted process $Y=\left\{Y_{t}, \mathscr{F}_{t} ; 0 \leq t<\infty\right\}$ is WSHB integrable with respect to stochastic process $X$, then the weakly Henstock variational belated integral of $Y$ is unique a.s.

In light of Proposition 8, we will denote the integral of the process $Y$ with respect to stochastic process $X$ by the notation

$$
\text { (WSH) } \int_{0}^{t} Y_{s} \circ d W_{s} \triangleq A_{t}
$$

Proposition 9. Let $Y$ and $Y^{\prime}=\left\{Y_{t}^{\prime}, \mathscr{F}_{t} ; 0 \leq t<\infty\right\}$ be WSHB integrable with respect to stochastic process $X$ and $\alpha \in \mathbb{R}$. Then
$Y+Y^{\prime}$ and $\alpha Y$ are WSHB integrable with respect to stochastic process $X$. Furthermore,

$$
\begin{aligned}
&(W S H) \int_{0}^{t}\left(Y_{s}+Y_{s}^{\prime}\right) \circ d X_{s}=(W S H) \int_{0}^{t} Y_{s} \circ d X_{s} \\
&+(W S H) \int_{0}^{t} Y_{s}^{\prime} \circ d X_{s} \\
&(W S H) \int_{0}^{t} \alpha Y_{s} \circ d X_{s}=\alpha(W S H) \int_{0}^{t} Y_{s} \circ d X_{s} .
\end{aligned}
$$

Proposition 10. If stochastic process $Y$ is WSHB integrable with respect to stochastic process $X$, then so is it on subinterval $[c, d] \subset[0, t]$.

Proposition 11. Let the adapted process $Y$ be WSHB integrable on $[0, a]$ and $[a, t]$ with respect to stochastic process $X$, where $0<a<t$. Then $Y$ is WSHB integrable on $[0, t]$ and, furthermore,

$$
\begin{aligned}
(W S H) \int_{0}^{t} Y_{s} \circ d X_{s}= & (W S H) \int_{0}^{a} Y_{s} \circ d X_{s} \\
& +(W S H) \int_{a}^{t} Y_{s} \circ d X_{s} .
\end{aligned}
$$

The proofs of Propositions 8 to 11 are similar to the results of classical Henstock integral [17]; hence we omit these proofs in this paper.

Next, we will consider the relationship between WSHB integral and Stratonovich integral with respect to continuous martingale. Here, we assume that the continuous martingale is bounded. In the next section we will address the continuous local martingale. Note that we can choose the stopping time such that a continuous local martingale becomes a bounded continuous martingale up to any finite stopping time [18, p. 44].

Theorem 12. If $f(x) \in C^{2}(\mathbb{R})$ and $X=\left\{X_{t}, \mathscr{F}_{t} ; 0 \leq t<\right.$ $\infty\}$ is bounded continuous martingale, then $f\left(X_{t}\right)$ is WSHB integrable with respect to bounded continuous martingale $X$ on $[0, t]$. Furthermore,

$$
(W S H) \int_{0}^{t} f\left(X_{s}\right) \circ d X_{s}=\int_{0}^{t} f\left(X_{s}\right) \circ d X_{s} .
$$

Proof. Since $X$ is a bounded continuous martingale and $f(x) \in C^{2}(\mathbb{R})$, then $f\left(X_{t}\right), f^{\prime}\left(X_{t}\right)$, and $f^{\prime \prime}\left(X_{t}\right)$ are bounded and continuous with respect to $t$. By Definition 1 , the classical Stratonovich integral with respect to bounded continuous martingale $X$ is

$$
\int_{0}^{t} f\left(X_{s}\right) \circ d X_{s}=\int_{0}^{t} f\left(X_{s}\right) d X_{s}+\int_{0}^{t} f^{\prime}\left(X_{s}\right) d\langle X\rangle_{s}
$$


Let $D_{1}=\left\{\left(\left[s_{i}, t_{i}\right], \xi_{i}\right)\right\}_{i=1}^{n}$ be a $(\delta, \eta)$-fine belated partial stochastic division of $[0, t]$. Then,

$$
\begin{aligned}
E \mid \sum_{i=1}^{n-1} \frac{1}{2}\left(f\left(X_{\xi_{i}}\right)+f\left(X_{\xi_{i+1}}\right)\right)\left(X_{t_{i}}-X_{s_{i}}\right) \\
-\left.\int_{s_{i}}^{t_{i}} f\left(X_{s}\right) \circ d X_{s}\right|^{2} \\
=E \mid \sum_{i=1}^{n-1} f\left(X_{\xi_{i}}\right)\left(X_{t_{i}}-X_{s_{i}}\right) \\
+\sum_{i=1}^{n-1} \frac{1}{2}\left(f\left(X_{\xi_{i+1}}\right)-f\left(X_{\xi_{i}}\right)\right)\left(X_{t_{i}}-X_{s_{i}}\right) \\
\quad-\left.\int_{s_{i}}^{t_{i}} f\left(X_{s}\right) \circ d X_{s}\right|^{2} \\
=E \mid \sum_{i=1}^{n-1} f\left(X_{\xi_{i}}\right)\left(X_{t_{i}}-X_{s_{i}}\right) \\
\quad+\sum_{i=1}^{n-1} \frac{1}{2}\left(f\left(X_{\xi_{i+1}}\right)-f\left(X_{\xi_{1}}\right)\right)\left(X_{t_{i}}-X_{s_{i}}\right) \\
\quad-\int_{s_{i}}^{t_{i}} f\left(X_{s}\right) d X_{s}-\left.\int_{s_{i}}^{t_{i}} \frac{\partial f\left(X_{s}\right)}{\partial x} d\langle X\rangle_{s}\right|^{2} \\
\leq 2 E\left|\sum_{i=1}^{n-1} f\left(X_{\xi_{i}}\right)\left(X_{t_{i}}-X_{s_{i}}\right)-\int_{s_{i}}^{t_{i}} f\left(X_{s}\right) d X_{s}\right|^{2} \\
+E \mid \sum_{i=1}^{n-1}\left(f\left(X_{\xi_{i+1}}\right)-f\left(X_{\xi_{i}}\right)\right)\left(X_{t_{i}}-X_{s_{i}}\right) \\
\quad-\left.\int_{s_{i}}^{t_{i}} \frac{\partial f\left(X_{s}\right)}{\partial x}\langle X\rangle_{s}\right|^{2}=2 R_{1}+R_{2}, \\
\hline
\end{aligned}
$$

where

$$
\begin{gathered}
R_{1}=E\left|\sum_{i=1}^{n-1} f\left(X_{\xi_{i}}\right)\left(X_{t_{i}}-X_{s_{i}}\right)-\int_{s_{i}}^{t_{i}} f\left(X_{s}\right) d X_{s}\right|^{2}, \\
R_{2}=E \mid \sum_{i=1}^{n-1} f\left(X_{\xi_{i+1}}\right)-f\left(X_{\xi_{i}}\right)\left(X_{t_{i}}-X_{s_{i}}\right) \\
\quad-\left.\int_{s_{i}}^{t_{i}} \frac{\partial f\left(X_{s}\right)}{\partial x} d\langle X\rangle_{s}\right|^{2} .
\end{gathered}
$$

From [12], given $\varepsilon_{1}>0$, there exists a $\left(\delta_{1}, \eta_{1}\right)$-fine belated partial division of $[0, t], D_{1}=\left\{\left(\left[s_{i}, t_{i}\right], \xi_{i}\right)\right\}_{i=1}^{n}$, such that $R_{1}<$ $\varepsilon_{1}$. We just need to consider $R_{2}$. By mean value theorem, $f\left(X_{\xi_{i+1}}\right)-f\left(X_{\xi_{i}}\right)=(\partial f / \partial x)\left(\widetilde{X_{i}}\right)\left(X_{\xi_{i+1}}-X_{\xi_{i}}\right)$, where $\widetilde{X}_{i} \epsilon$ $\left[X_{\xi_{i}}, X_{\xi_{i+1}}\right]$. Since $X_{t}$ is continuous with respect to $t$, there exists a $\bar{\xi}_{i} \in\left[\xi_{i}, \xi_{i+1}\right]$ such that $X_{\widetilde{\xi}_{i}}=\widetilde{X}_{i}$ a.s. Similarly, there exists $\bar{\xi}_{i} \in\left[s_{i}, t_{i}\right]$ such that $\int_{s_{i}}^{t_{i}}\left(\partial f\left(X_{t}\right) / \partial x\right) d\langle X\rangle_{t}=$ $f^{\prime}\left(X_{\bar{\xi}_{i}}\right)\left(X_{t_{i}}-X_{s_{i}}\right)^{2}$ in $L^{2}(\Omega)$ space. Then,

$$
\begin{gathered}
R_{2}=E \mid \sum_{i=1}^{n-1}\left(f\left(X_{\xi_{i+1}}\right)-f\left(X_{\xi_{i}}\right)\right)\left(X_{t_{i}}-X_{s_{i}}\right) \\
\quad-\left.\int_{s_{i}}^{t_{i}} \frac{\partial f\left(X_{t}\right)}{\partial x} d\langle X\rangle_{t}\right|^{2} \\
=E \mid \sum_{i=1}^{n-1}\left\{f^{\prime}\left(X_{\bar{\xi}_{i}}\right)\left(X_{\xi_{i+1}}-X_{\xi_{i}}\right)\left(X_{t_{i}}-X_{s_{i}}\right)\right. \\
\leq 2 E \mid \sum_{i=1}^{n-1}\left\{f^{\prime}\left(X_{\bar{\xi}_{i}}\right)\left(X_{\xi_{i+1}}-X_{\xi_{i}}\right)\left(X_{t_{i}}-X_{s_{i}}\right)\right. \\
\left.-\frac{\partial f\left(X_{\bar{\xi}_{i}}\right)}{\partial x}\left(X_{t_{i}}-X_{s_{i}}\right)^{2}\right\}\left.\right|^{2} \\
\left.+2 E \mid \sum_{i=1}^{n-1} f^{\prime}\left(X_{\bar{\xi}_{i}}\right)\left(X_{\bar{\xi}_{i}}-X_{s_{i}}\right)^{2}\left(X_{t_{i}}-X_{s_{i}}\right)^{2}\right\}\left.\right|^{2} \\
-\left.f^{\prime}\left(X_{\bar{\xi}_{i}}\right)\left(X_{t_{i}}-X_{s_{i}}\right)^{2}\right|^{2} .
\end{gathered}
$$

Let

$$
\begin{gathered}
r_{1}=E \mid \sum_{i=1}^{n-1}\left\{f^{\prime}\left(X_{\widehat{\xi}_{i}}\right)\left(X_{\xi_{i+1}}-X_{\xi_{i}}\right)\left(X_{t_{i}}-X_{s_{i}}\right)\right. \\
\left.-f^{\prime}\left(X_{\bar{\xi}_{i}}\right)\left(X_{t_{i}}-X_{s_{i}}\right)^{2}\right\}\left.\right|^{2},
\end{gathered}
$$

$$
\begin{aligned}
r_{2}= & E\left|\sum_{i=1}^{n-1} f^{\prime}\left(X_{\bar{\xi}_{i}}\right)\left(X_{t_{i}}-X_{s_{i}}\right)^{2}-f^{\prime}\left(X_{\bar{\xi}_{i}}\right)\left(X_{t_{i}}-X_{s_{i}}\right)^{2}\right|^{2}, \\
r_{1} & \leq K E\left|\sum_{i=1}^{n-1}\left(X_{\xi_{i+1}}-X_{\xi_{i}}\right)\left(X_{t_{i}}-X_{s_{i}}\right)-\left(X_{t_{i}}-X_{s_{i}}\right)^{2}\right|^{2} \\
& \leq K E\left|\sum_{i=1}^{n-1}\left(X_{t_{i}}-X_{s_{i}}\right)\left[\left(X_{t_{i}}-X_{s_{i}}\right)-\left(X_{\xi_{i+1}}-X_{\xi_{i}}\right)\right]\right|^{2} \\
& \leq K E\left|\sum_{i=1}^{n-1}\left(X_{t_{i}}-X_{s_{i}}\right)\left[\left(X_{\xi_{i+1}}-X_{t_{i}}\right)+\left(X_{s_{i}}-X_{\xi_{i}}\right)\right]\right|^{2} \\
& \leq K \sum_{i=1}^{n-1} E \mid\left(X_{\xi_{i+1}}-X_{t_{i}}\right)+\left(X_{s_{i}}-\left.X_{\xi_{i}}\right|^{2} E\left(X_{t_{i}}-X_{s_{i}}\right)^{2},\right.
\end{aligned}
$$


where $K=\sup \left\{f^{\prime}\left(W_{t}\right): 0 \leq t \leq 1 \& \omega \in \Omega\right\}$. Given that $X_{t}$ is a bounded martingale,

$$
\begin{aligned}
& E\left|\left(X_{\xi_{i+1}}-X_{t_{i}}\right)+\left(X_{s_{i}}-X_{\xi_{i}}\right)\right|^{2} \\
& \quad=E\left(X_{\xi_{i+1}}-X_{t_{i}}\right)^{2}+E\left(X_{s_{i}}-X_{\xi_{i}}\right)^{2} \\
& \quad=E\langle X\rangle_{\xi_{i+1}}-E\langle X\rangle_{t_{i}}+E\langle X\rangle_{s_{i}}-E\langle X\rangle_{\xi_{i}} .
\end{aligned}
$$

However, there exists a $\left(\delta_{2}, \eta_{2}\right)$-fine belated partial stochastic division $\left.D_{2}=\left\{\left[\xi_{i}, t_{i}\right], \xi_{i}\right): i=1,2, \ldots, n\right\}$ of $[0, t]$ such that $\left|\mu_{X}(0, t]-\sum_{i=1}^{n} \mu_{X}\left(s_{i}, t_{i}\right]\right|<\eta_{2}$. Then we have

$$
E\langle X\rangle_{\xi_{i+1}}-E\langle X\rangle_{t_{i}}+E\langle X\rangle_{s_{i}}-E\langle X\rangle_{\xi_{i}}<\eta_{2} .
$$

Hence,

$$
r_{1} \leq K \varepsilon_{2} \sum_{i=1}^{n-1} E\left(X_{t_{i}}-X_{s_{i}}\right)^{2} \leq K E\langle X\rangle_{t} \eta_{2}
$$

Since $X$ is bounded continuous martingale, by Lemma 5.10 in [18, p. 33], we have

$$
E\left|\sum_{i=1}^{n-1}\left(X_{t_{i}}-X_{s_{i}}\right)^{4}\right| \longrightarrow 0 \quad \text { as } n \longrightarrow \infty .
$$

Hence, given $\varepsilon_{2}>0$,

$$
\begin{aligned}
r_{2} & \leq \sup _{1 \leq i \leq n \& \omega \in \Omega}\left|f^{\prime}\left(X_{\bar{\xi}_{i}}\right)-f^{\prime}\left(X_{\bar{\xi}_{i}}\right)\right| E\left|\sum_{i=1}^{n-1}\left(X_{t_{i}}-X_{s_{i}}\right)^{4}\right| \\
& \leq 2 K \varepsilon_{2} .
\end{aligned}
$$

Now take $\eta=\min \left\{\eta_{1}, \eta_{2}\right\}$ and let $\delta\left(\xi_{i}\right)=\min \left\{\delta_{1}\left(\xi_{i}\right), \delta_{2}, \xi_{i+1}-\right.$ $\left.\xi_{i}\right\}$; then a $(\delta, \eta)$-fine belated partial division $D=\left\{\left[\xi_{i}, t_{i}\right], \xi_{i}\right)$ : $i=1,2, \ldots, n\}$ of $[0, t]$ is both $\left(\delta_{1}, \eta_{1}\right)$-fine belated partial stochastic division and $\left(\delta_{2}, \eta_{2}\right)$-fine belated partial stochastic division. Hence, for $\varepsilon=\max \left\{\varepsilon_{1}, \eta_{2}, \varepsilon_{2}\right\}$, we have

$$
\begin{aligned}
& E\left|\sum_{i=1}^{n-1} \frac{f_{\xi_{i}}+f_{\xi_{i+1}}}{2}\left(W_{t_{i}}-W_{s_{i}}\right)-\int_{s_{i}}^{t_{i}} X_{t} \circ d W_{t}\right|^{2} \\
& \quad \leq R_{1}+r_{1}+r_{2} \leq \bar{K} \varepsilon,
\end{aligned}
$$

where $\bar{K}$ is a constant. Hence, $f\left(X_{t}\right)$ is WSHB integrable on $[0, t]$ and, by the unique property of WSHB,

$$
\text { (WSH) } \int_{0}^{t} f\left(X_{s}\right) \circ d X_{s}=\int_{0}^{t} f\left(X_{s}\right) \circ d X_{s} \text {. }
$$

\subsection{Henstock Approach to Define Stratonovich Integral with respect to Continuous Local Martingale}

Lemma 13. Let $X=\left\{X_{t}, \mathscr{F}_{t} ; 0 \leq t<\infty\right\}$ be a continuous local martingale. Suppose $f$ is WSHB integrable to a process $A_{t}$ with respect to local continuous martingale $X$. Let $T$ be a stopping time; then $f$ is WSHB integrable to a process $A^{T}$ with respect to $X^{T}$, where $X^{T}=\left\{X_{t \wedge T} ; 0 \leq t<\infty\right\}$ and $A^{T}=$ $\left\{A_{t \wedge T} ; 0 \leq t<\infty\right\}$.
Proof. Let $\varepsilon>0$ and $\delta(\xi, \omega)>0$ be given as in Definition 6 for the WSHB integral of $f$ with respect to continuous local martingale $X$. Define

$$
\delta^{T}(\xi, \omega) \triangleq \begin{cases}T(\omega)-\xi, & \text { if } \xi<T(\omega) \leq \xi+\delta(\xi, \omega) \\ \delta(\xi, \omega), & \text { otherwise. }\end{cases}
$$

Then $\delta^{T}(\xi, \omega)$ is locally stopping process, since $(T(\omega) \wedge \xi) \wedge$ $(\xi+\delta(\xi, \omega))$ is a stopping time and $\{\omega: T(\omega)<\xi\} \in \mathscr{F}_{t}$ if $\xi<t$. Let $((U, V], \xi)$ be a $\left(\delta^{T}(\xi, \omega), \eta\right)$-fine belated stochastic interval-point pair.

(i) If $T(w) \leq \xi$, then

$$
\begin{aligned}
X^{T} & (V(\omega), \omega)-X^{T}(U(\omega), \omega) \\
& =X^{T}(T(\omega), \omega)-X^{T}(T(\omega), \omega)=0 .
\end{aligned}
$$

(ii) If $\xi<T(\omega) \leq \xi+\delta(\xi, \omega)$, then $[U, V] \subset[\xi, T] \subset$ $[\xi, \xi+\delta(\xi, \omega)]$. Consequently, $((U, V], \xi)$ will be a $(\delta(\xi, \omega), \eta)$-fine belated stochastic interval-point pair, $U \wedge T=U$ and $V \wedge T=V$.

(iii) If $T(\omega)>\xi+\delta(\xi, \omega)$, then

$$
\begin{aligned}
X^{T} & (U(\omega), \omega)-X^{T}(V(\omega), \omega) \\
& =X(V(\omega) \wedge T(\omega), \omega)-X(U(\omega) \wedge T(\omega), \omega) \\
& =X(U(\omega), \omega)-X(V(\omega), \omega) .
\end{aligned}
$$

It is easy to verify that $D=\left\{\left(\left(U_{i}, V_{i}\right], \xi_{i}\right), i=1,2, \ldots, n\right\}$ is also a $\left(\delta^{T}(\xi, \omega), \eta\right)$-fine belated stochastic division of $[0, t \wedge T(w)]$ only if $D=\left\{\left(\left(U_{i}, V_{i}\right], \xi_{i}\right), i=1,2, \ldots, n\right\}$ is a $(\delta(\xi, \omega), \eta)$-fine belated stochastic division of $[0, t]$.

Similar results hold for $A^{T}$. Since the $D=$ $\left\{\left(\left(U_{i}, V_{i}\right], \xi_{i}\right), i=1,2, \ldots, n\right\}$ is also a $\left(\delta^{T}(\xi, \omega), \eta\right)$-fine belated stochastic division of $[0, t \wedge T(w)]$, by the property of being WSHB integrable, we have that $f$ is WSHB integrable to a process $A^{T}$ with respect to $X^{T}$.

Lemma 14. Let $X=\left\{X_{t}, \mathscr{F}_{t} ; 0 \leq t<\infty\right\}$ be a continuous local martingale with the corresponding nondecreasing sequence of stopping times $\left\{T_{n}\right\}_{n=1}^{\infty}$. Suppose that $f$ is WSHB integrable to a process $A$ with respect to $X$; then $f$ is WSHB integrable to a process $A^{T_{n}}$ with respect to $X^{T_{n}}$ for each $n=$ $1,2, \ldots$. Furthermore, for each $t \in[0, t], \lim _{n \rightarrow \infty} A^{T_{n}}=A$ a.s.

Proof. This follows directly form Lemma 13 and the uniqueness of the WSHB integral. Then we have the last statement

$$
\lim _{n \rightarrow \infty} A^{T_{n}}=A \text { a.s. }
$$

Theorem 15. If $f(x) \in C^{2}(\mathbb{R})$ and $X=\left\{X_{t}, \mathscr{F}_{t} ; 0 \leq t<\infty\right\}$ is a continuous local martingale, then $f\left(X_{t}\right)$ is WSHB integrable with respect to $X$ on $[0, t]$. Furthermore,

$$
(W S H) \int_{0}^{t} f\left(X_{s}\right) \circ d X_{s}=\int_{0}^{t} f\left(X_{s}\right) \circ d X_{s} .
$$


Proof. First we introduce, for each $m \geq 1$, the stopping time

$$
T_{m}=\inf \left\{t \geq 0 ;\left|X_{t}\right| \geq m \text { or }\langle X\rangle_{t} \geq m\right\}
$$

The resulting sequence $\left\{T_{m}\right\}$ is nondecreasing with $P\left[\lim _{m \rightarrow \infty} T_{m}=\infty\right]=1$. Thus, the stopping process $X_{t \wedge T_{m}}$ is bounded; that is, $X_{t \wedge T_{m}}$ is a bounded martingale for each $m=1,2, \ldots$. Under this situation, the values of $f$ outside $[-m, m]$ are irrelevant. We may assume without loss of generality that $f$ has compact support, so $f, f^{\prime}$, and $f^{\prime \prime}$ are bounded.

Let $D=\left\{\left(\left(U_{i}, V_{i}\right], \xi_{i}\right), i=1,2, \ldots, n\right\}$ be a $(\delta(\xi, \omega), \eta)$-fine belated stochastic division of $[0,1]$. From Lemma 13, we have that, for each $m \geq 1, D_{m}=\left\{\left(\left(U_{i}, V_{i}\right], \xi_{i}\right), i=1,2, \ldots, n\right\}$ is a $\left(\delta^{T_{m}}(\xi, \omega), \eta\right)$-fine belated stochastic division of $\left[0, t \wedge T_{m}(w)\right]$. From Lemma 13, there is a process $A^{T_{m}}=\left\{A_{t \wedge T_{m}} ; 0 \leq t<\infty\right\}$ such that

$$
\begin{gathered}
E\left(\mid \sum_{i=1}^{n-1} \frac{1}{2} f\left(X_{\xi_{i+1}}+X_{\xi_{i}}\right)\left(X_{V_{i} \wedge T_{m}}-X_{U_{i} \wedge T_{m}}\right)\right. \\
\left.-\left.\left(A_{V_{i} \wedge T_{m}}-A_{U_{i} \wedge T_{m}}\right)\right|^{2}\right)<\varepsilon,
\end{gathered}
$$

for every $(\delta, \eta)$-fine belated partial stochastic division $D=$ $\left\{\left(\left(U_{i}, V_{i}\right], \xi_{i}\right): i=1,2, \ldots, n\right\}$ of $[0, t]$. Since

$$
\begin{aligned}
\lim _{m \rightarrow \infty} \mid & \mid \sum_{i=1}^{n-1} f\left(X_{\xi_{i+1}}+X_{\xi_{i}}\right)\left(X_{V_{i} \wedge T_{m}}-X_{U_{i} \wedge T_{m}}\right) \\
& -\left(A_{V_{i} \wedge T_{m}}-A_{U_{i} \wedge T_{m}}\right) \mid \\
=\mid \sum_{i=1}^{n-1} f\left(X_{\xi_{i+1}}+X_{\xi_{i}}\right)\left(X_{V_{i}}-X_{U_{i}}\right) & \\
& -\left(A_{V_{i}}-A_{U_{i}}\right) \mid \text { a.s., }
\end{aligned}
$$

we must have

$$
\begin{aligned}
& E\left|\sum_{i=1}^{n-1} f\left(X_{\xi_{i+1}}+X_{\xi_{i}}\right)\left(X_{V_{i}}-X_{U_{i}}\right)-\left(A_{V_{i}}-A_{U_{i}}\right)\right| \\
& \quad=E\left(\lim _{m \rightarrow \infty} \mid \sum_{i=1}^{n-1} \frac{1}{2} f\left(X_{\xi_{i+1}}+X_{\xi_{i}}\right)\left(X_{V_{i} \wedge T_{m}}-X_{U_{i} \wedge T_{m}}\right)\right.
\end{aligned}
$$

$$
\begin{gathered}
\left.-\left(A_{V_{i} \wedge T_{m}}-A_{U_{i} \wedge T_{m}}\right) \mid\right)^{2} \\
=E\left(\liminf _{m \rightarrow \infty} \mid \sum_{i=1}^{n-1} \frac{1}{2} f\left(X_{\xi_{i+1}}+X_{\xi_{i}}\right)\left(X_{V_{i} \wedge T_{m}}-X_{U_{i} \wedge T_{m}}\right)\right. \\
\left.-\left(A_{V_{i} \wedge T_{m}}-A_{U_{i} \wedge T_{m}}\right) \mid\right)^{2} \\
\leq \liminf _{m \rightarrow \infty}\left(\mid \sum_{i=1}^{n-1} \frac{1}{2} f\left(X_{\xi_{i+1}}+X_{\xi_{i}}\right)\left(X_{V_{i} \wedge T_{m}}-X_{U_{i} \wedge T_{m}}\right)\right. \\
\left.-\left(A_{V_{i} \wedge T_{m}}-A_{U_{i} \wedge T_{m}}\right) \mid\right)^{2} \\
<\liminf _{m \rightarrow \infty} \leq \varepsilon .
\end{gathered}
$$

This shows that $f\left(X_{t}\right)$ is WSHB integrable on $[0, t]$ and

$$
\text { (WSH) } \int_{0}^{t} f\left(X_{s}\right) \circ d X_{s}=\int_{0}^{t} f\left(X_{s}\right) \circ d X_{s} \text {. }
$$

3.3. Henstock Approach to Define Stratonovich Integral with respect to Continuous Semimartingale. Let $X=\left\{X_{t}, \mathscr{F}_{t} ; 0 \leq\right.$ $t<\infty\}$ be a continuous semimartingale such that

$$
X_{t}=X_{0}+M_{t}+C_{t}
$$

where $M_{t}$ is a continuous local martingale and $C_{t}$ is adapted, continuous process of bounded variation with $C_{0}=0$ a.s. We have

$$
\begin{aligned}
\frac{1}{2} f( & \left.X_{\xi_{i+1}}+X_{\xi_{i}}\right)\left(X_{V_{i}}-X_{U_{i}}\right) \\
= & \frac{1}{2} f\left(X_{\xi_{i+1}}+X_{\xi_{i}}\right)\left(M_{V_{i}}-M_{U_{i}}\right) \\
& +\frac{1}{2} f\left(X_{\xi_{i+1}}+X_{\xi_{i}}\right)\left(C_{V_{i}}-C_{U_{i}}\right) .
\end{aligned}
$$

Now we consider a stopping time

$$
T_{m}=\left\{\begin{array}{l}
\text { if } \quad\left|X_{0}\right| \geq m \\
\text { inf }\left\{t \geq 0 ;\left|M_{t}\right| \geq m\right. \text { or } \\
\left.\langle M\rangle_{t} \geq m \text { or } \breve{C}_{t} \geq m\right\} \\
\quad \text { if }\left|X_{0}\right|<m \\
\quad \text { if }\left|X_{0}\right|<m \text { \& } \\
\quad \inf \left\{t \geq 0 ;\left|M_{t}\right| \geq m\right. \text { or } \\
\left.\quad\langle M\rangle_{t} \geq m \text { or } \breve{C}_{t} \geq m\right\}=\infty
\end{array}\right.
$$

where $\breve{C}_{t}$ is the total variation of $C_{t}$ on $[0, t]$. Then the sequence $\left\{T_{m}\right\}$ is nondecreasing with $P\left[\lim _{m \rightarrow \infty} T_{m}=\infty\right]=$ 1. Thus, the stopping process $M_{t \wedge T_{m}}$ is a bounded martingale 
for each $m=1,2, \ldots$ In addition, $C_{t} 1_{\left\{t \leq T_{m}(w)\right\}}$ is of bounded variation. Under this situation, the values of $f$ outside $[-3 m, 3 m]$ are irrelevant. We may assume without loss of generality that $f$ has compact support, so $f, f^{\prime}$, and $f^{\prime \prime}$ are bounded. Given that the $\sum_{i=1}^{n}(1 / 2) f\left(X_{\xi_{i+1}}+X_{\xi_{i}}\right)\left(C_{V_{i}}-C_{U_{i}}\right)$ is classic Riemann-Stieltjes integral, $(1 / 2) f\left(X_{\xi_{i+1}}+X_{\xi_{i}}\right)\left(A_{V_{i}}-\right.$ $\left.A_{U_{i}}\right)$ is WSHB integrable for every $(\delta, \varepsilon)$-fine belated partial stochastic division $D=\left\{\left(\left(U_{i}, V_{i}\right], \xi_{i}\right): i=1,2, \ldots, n\right\}$ of $[0, t]$ (see [17]). Since the WSHB integral satisfies the linear property, we obtain the following result.

Theorem 16. If $f(x) \in C^{2}(\mathbb{R})$ and $X$ is a continuous semimartingale, then $f\left(X_{t}\right)$ is WSHB integrable with respect to $X_{t}$ on $[0, t]$. Furthermore,

$$
(W S H) \int_{0}^{t} f\left(X_{s}\right) \circ d X_{s}=\int_{0}^{t} f\left(X_{s}\right) \circ d X_{s} .
$$

\section{Conclusion}

From Theorem 16, if $f$ is of class $C^{2}(\mathbb{R})$ and $X$ is a continuous semimartingale, $f\left(X_{t}\right)$ is WSHB integrable to $\int_{0}^{t} f\left(X_{s}\right) \circ d X_{s}$ on $[0, t]$. Now we consider the Itô formula for Stratonovich integral; if $f \in C^{3}(\mathbb{R})$, as shown in Proposition 4,

$$
f\left(X_{t}\right)=f\left(X_{0}\right)+\int_{0}^{t} f^{\prime}\left(X_{s}\right) \circ d X_{s} .
$$

We substitute (WSH) $\int_{0}^{t} f^{\prime}\left(X_{s}\right) \circ d X_{s}$ with $\int_{0}^{t} f^{\prime}\left(X_{s}\right) \circ d X_{s}$. Then,

$$
f\left(X_{t}\right)=f\left(X_{0}\right)+(\mathrm{WSH}) \int_{0}^{t} f^{\prime}\left(X_{s}\right) \circ d X_{s} .
$$

In conclusion, from the definition of Stratonovich integral with respect to continuous semimartingale using Henstock method, we manage to keep the important properties of the classical Stratonovich integral and also probably enlarge the scope of the integrands which satisfy classical Stratonovich integral Itô formula (46).

\section{Conflict of Interests}

The authors declare that there is no conflict of interests regarding the publication of this paper.

\section{References}

[1] P. Protter, Stochastic Integration and Differential Equations, Springer, New York, NY, USA, 2nd edition, 2004.

[2] M. Gradinaru, I. Nourdin, F. Russo, and P. Vallois, "m-order integrals and generalized Itô's formula; the case of a fractional Brownian motion with any Hurst index," Annales de l'institut Henri Poincare (B) Probability and Statistics, vol. 41, no. 4, pp. 781-806, 2005.

[3] B. Øksendal, Stochastic Differential Equations, Springer, 6th edition, 2005.

[4] R. Henstock, Lectures on the Theory of Integration, vol. 1 of Series in Real Analysis, World Scientific, Singapore, 1988.
[5] R. Henstock, "The efficiency of convergence factors for functions of a continuous real variable," Journal of the London Mathematical Society, vol. 30, pp. 273-286, 1955.

[6] J. Kurzweil, "Generalized ordinary differential equations and continuous dependence on a parameter," Czechoslovak Mathematical Journal, vol. 7, no. 3, pp. 418-449, 1957.

[7] R. Henstock, The General Theory of Integration, Oxford University Press, Oxford, UK, 1991.

[8] D. Nualart, The Malliavin Calculus and Related Topics, Springer, New York, NY, USA, 1995.

[9] D. Nualart and E. Pardoux, "Stochastic calculus with anticipating integrands," Probability Theory and Related Fields, vol. 78, no. 4, pp. 535-581, 1988.

[10] P. Protter, "A comparison of stochastic integrals," The Annals of Probability, vol. 7, no. 2, pp. 276-289, 1979.

[11] R. L. Stratonovich, "A new representation for stochastic integrals and equations," SIAM Journal on Control and Optimization, vol. 4, pp. 362-371, 1966.

[12] T.-L. Toh and T.-S. Chew, "The Riemann approach to stochastic integration using non-uniform meshes," Journal of Mathematical Analysis and Applications, vol. 280, no. 1, pp. 133-147, 2003.

[13] H. F. Yang and T. L. Toh, "On Henstock-Kurzweil method to Stratonovich integral," Mathematical Bohemica, In press.

[14] T. L. Toh and T. S. Chew, "A variational approach to Itô's integral," in Proceedings of SAP's '98, pp. 291-299, 1998.

[15] T.-L. Toh and T.-S. Chew, "The Kurzweil-Henstock theory of stochastic integration," Czechoslovak Mathematical Journal, vol. 62, no. 3, pp. 829-848, 2012.

[16] T.-S. Chew, J.-Y. Tay, and T.-L. Toh, "The non-uniform Riemann approach to Itô's integral," Real Analysis Exchange, vol. 27, no. 2, pp. 495-514, 2001.

[17] P. Y. Lee, Lanzhou Lectures on Henstock Integration, World Scientific, Singapore, 1989.

[18] I. Karatzas and S. E. Shreve, Brownian Motion and Stochastic Calculus, Springer, New York, NY, USA, 2nd edition, 2000.

[19] E. J. McShane, Stochastic Calculus and Stochastic Models, Academic Press, 1974.

[20] T.-L. Toh and T.-S. Chew, "On Iô-Kurzweil-Henstock integral and integration-by-part formula," Czechoslovak Mathematical Journal, vol. 55, no. 3, pp. 653-663, 2005. 


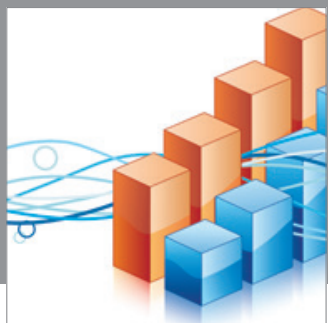

Advances in

Operations Research

mansans

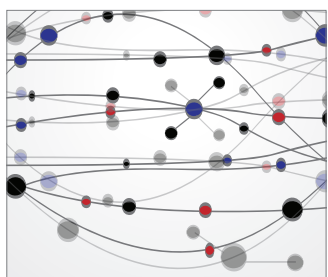

The Scientific World Journal
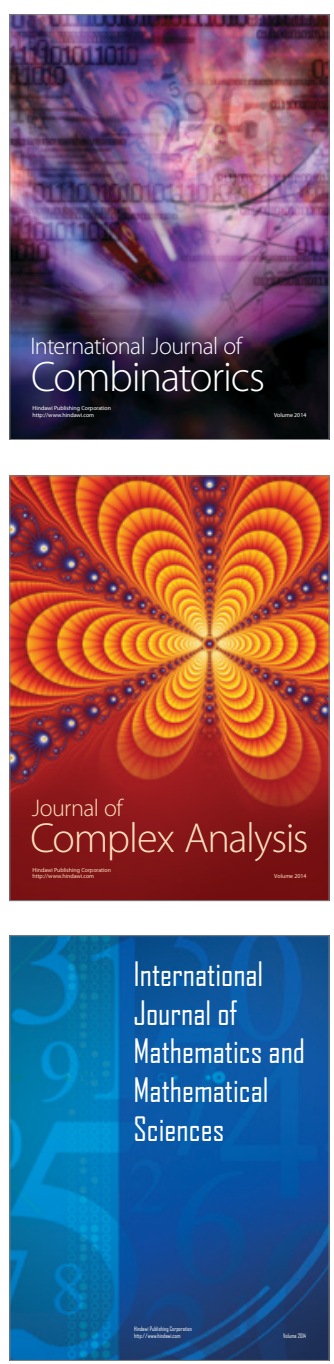
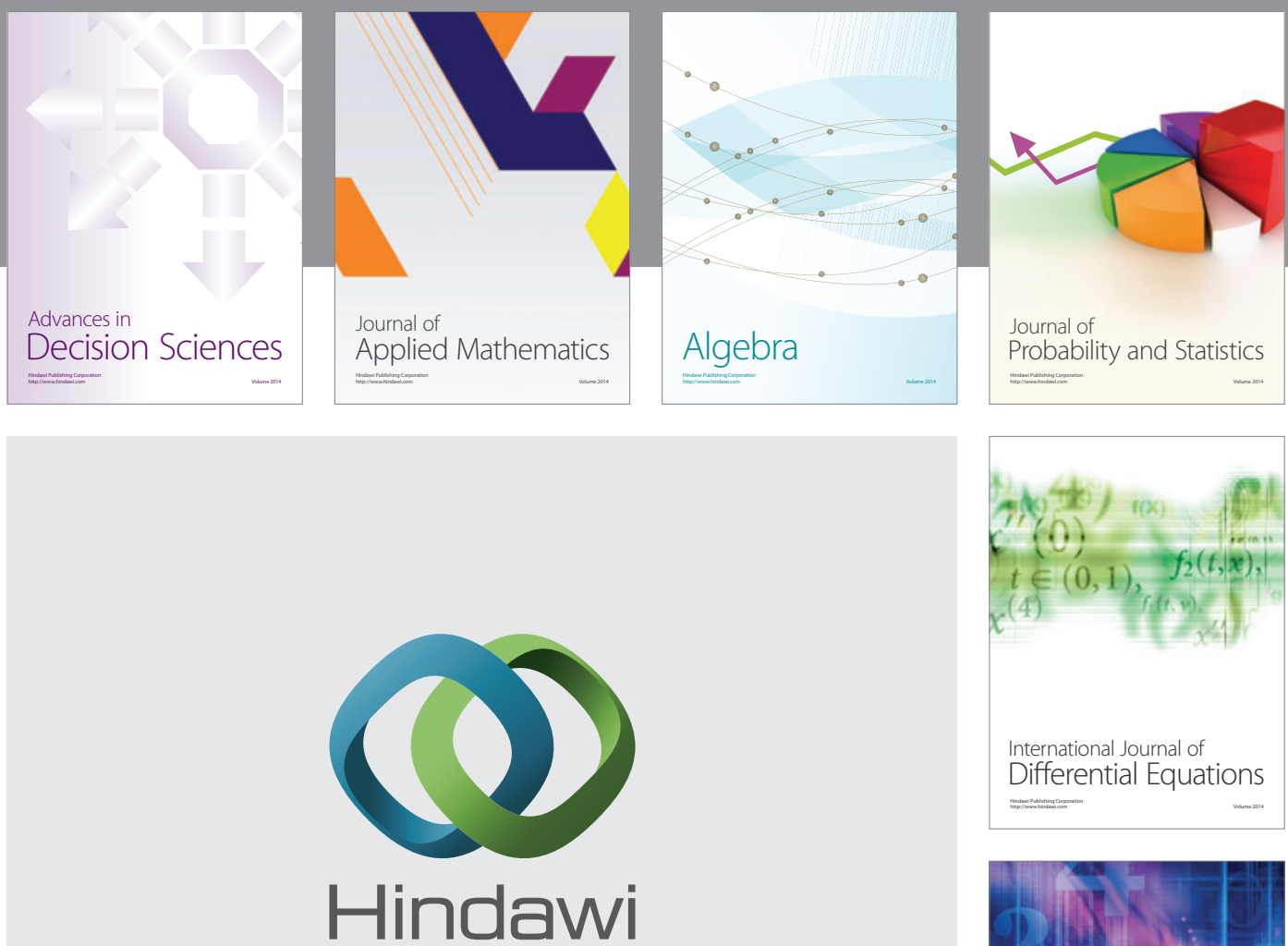

Submit your manuscripts at http://www.hindawi.com
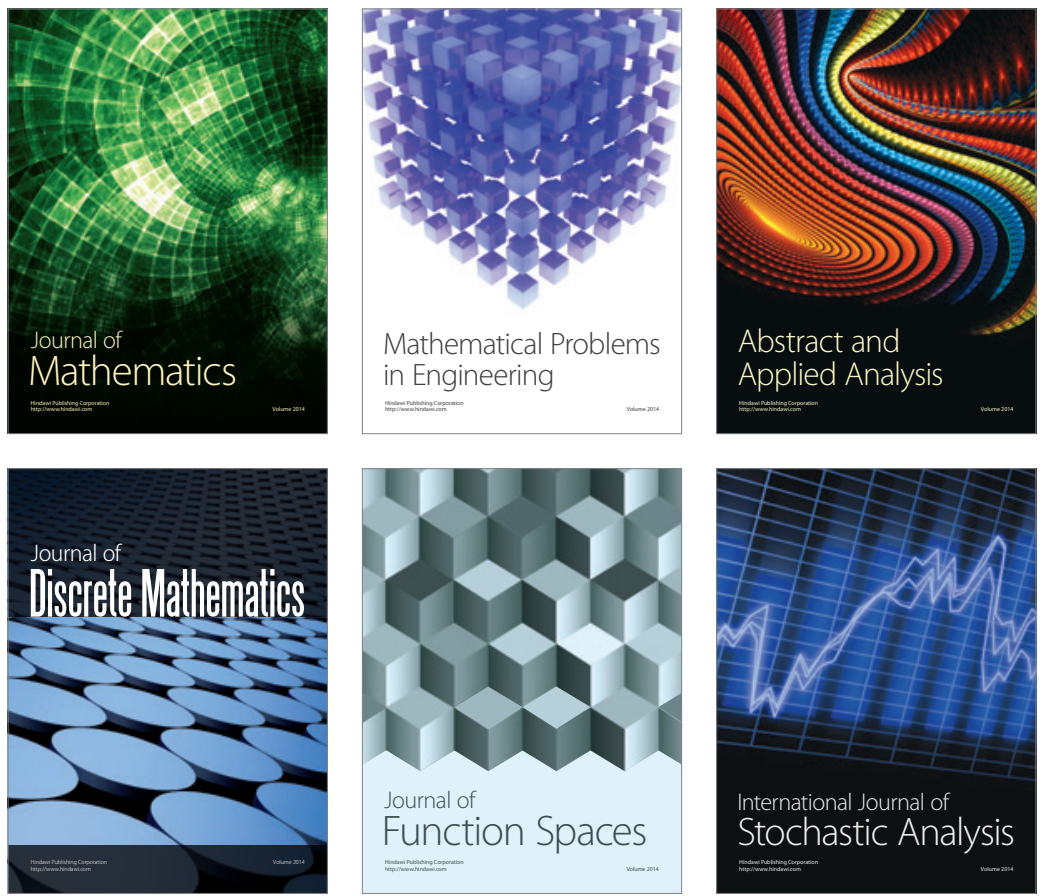

Journal of

Function Spaces

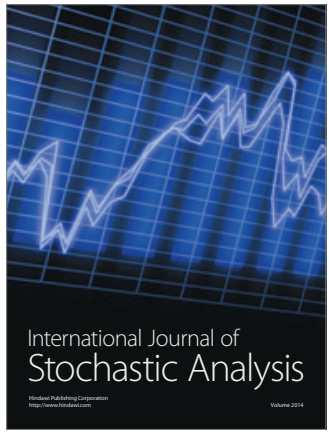

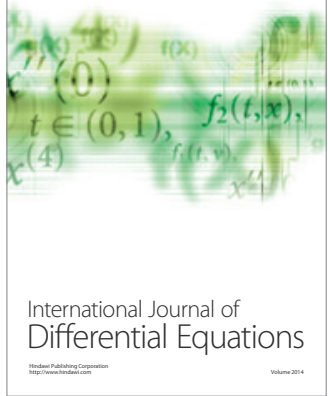
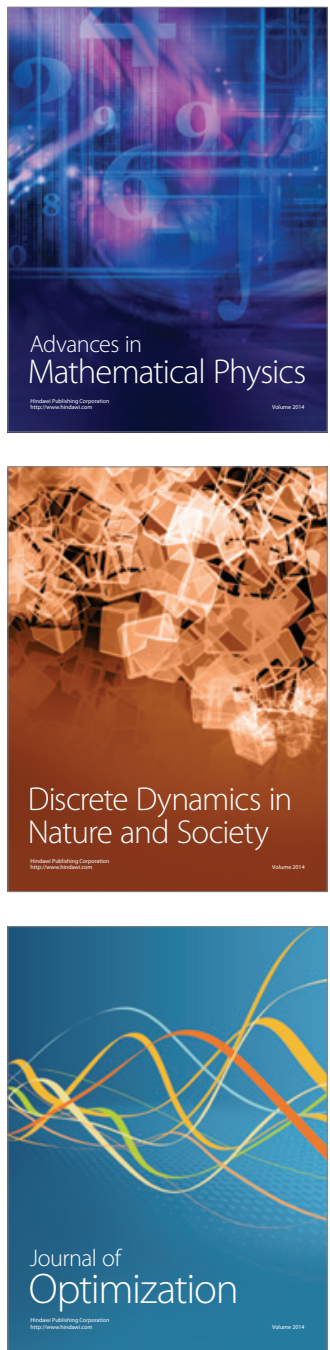